\title{
Preparing for Accreditation Under EC 2000: An Experience Report
}

\author{
Neelam Soundarajan* \\ Computer \& Information Science Department \\ College of Engineering, Ohio State University \\ Columbus, OH 43210 \\ e-mail: neelam@cis.ohio-state.edu
}

\begin{abstract}
By now it is well understood in the engineering community that preparing for accreditation evaluation under Engineering Criteria 2000 is a demanding task. Perhaps the most challenging requirements of the criteria have to do with setting up mechanisms to obtain input from various constituencies, formulating precise objectives and outcomes on the basis of these inputs, setting up a range of assessment mechanisms to evaluate how successful the program is in meeting these objectives and outcomes, and setting up feedback mechanisms to use the results of the assessments to improve the program. This paper presents the experience of one program in doing this, and some of the lessons learned.
\end{abstract}

\section{Introduction}

By now it is well understood in the engineering community that preparing for accreditation evaluation under Engineering Criteria 2000 (henceforth abbreviated EC 2000) is a demanding task $[4,8,6]$. Perhaps the most challenging of the EC 2000 requirements are those that have to do with objectives, outcomes, and assessments. Traditionally, most engineering programs have not paid much explicit attention to these issues, focusing instead on curricular questions [8]. This was certainly the case with the Computer Science and Engineering (CSE) program in the Computer and Information Science (CIS) Department at Ohio State until about three years ago when the decision was made to seek evaluation under EC 2000. As chair of the Undergraduate Studies Committee of the department, I was charged with leading the

*The opinions expressed in this article are those of the author as an individual educator and researcher. They do not reflect the official position of the Ohio State University, its College of Engineering, or the Computer \& Information Science Dept. 
effort to get the program ready for the evaluation. In this paper, I summarize how we ${ }^{1}$ approached the task, describe some of the mechanisms we implemented to obtain input from constituencies and for assessment, and try to draw some general lessons from our experience; I also try to relate our experiences to those of other programs as reported in the literature.

The main requirements of EC 2000 related to objectives, outcomes, etc., may be summarized as follows:

1. Develop processes and mechanisms to collect input from various constituencies such as current students, graduates, local industries, etc., on various aspects of the program.

2. Determine objectives and outcomes for the program based on the needs of the constituents, and publish the objectives and outcomes; set up processes to periodically evaluate and improve the objectives and outcomes, based on constituent inputs.

3. Set up assessment mechanisms to measure the achievement of these objectives and outcomes, as well as the outcomes listed in Criterion 3 of EC 2000; and use the results of the assessments to improve the program.

4. Document these processes and assessment mechanisms and their application; and document the improvements in the program resulting from the assessments.

In Section 2, I describe the mechanisms we set up to collect input from our constituents, and detail the process we used in arriving at our objectives and outcomes; I also briefly discuss potential problems in the input collection process, and relate these to the approach we adopted. In Section 3, I summarize the objectives and outcomes we established for the program, and suggest an enhancement to the criteria that will enable engineering programs to borrow the best ideas from other programs, rather than each one starting from scratch. In Section 4, I summarize the assessment mechanisms we have set up to evaluate achievement of these objectives and outcomes; I also discuss potential problems behind some assessment mechanisms. Section 5 summarizes the lessons that may be drawn from the experience of our program.

This paper makes two contributions. First, for programs gearing up for evaluation under EC 2000 in the next year or two, it should provide some ideas on how to meet the requirements and what some of the potential problems may be. Second, I hope it will contribute ideas for possible refinements and improvements to the accreditation criteria themselves.

\section{Constituent Inputs}

Criterion 2 of EC 2000 requires, in part, that the program must have in place "a process based on the needs of the program's various constituencies in which the objectives are determined and periodically evaluated". The first step in meeting this requirement is to identify the constituencies. For most programs the main constituencies would be current students, alumni, and (current and prospective) employers of graduates of the program; graduate

\footnotetext{
${ }^{1}$ Throughout the paper, 'I, my' etc. refer to the author; 'we, our' etc. refer to the faculty of the CIS Department as a whole.
} 
schools might also be considered a constituency depending on the percentage of the graduates of the program who go to graduate school.

There are two questions to consider in meeting this criterion. First is the question of how to determine the needs of the constituencies so that appropriate objectives can be determined. Aldridge and Benefield [2] caution "Educators must ensure that the results [of the processes used to determine constituency needs] are not biased in favor of their own preconceptions about constituency needs". But then, continuing in the same article, they note, "... this type of information gathering tends to identify a group's perceived needs and expectations, not necessarily its real or future needs"! Clearly this is no easy task.

Another angle on this problem is presented by Sener [9]. Sener reports on the results of asking individuals on their industrial advisory board to rank order the importance of a set of eleven proposed objectives which were based directly on the abilities listed in Criterion 3 of EC 2000. Remarkably, there was almost no consistency in the ordering that different members of this group came up with for the proposed objectives. For example, the objective related to the ability to design and conduct experiments and to analyze data, received rankings ranging from the highest ranking of one through dead last at eleven. Even the objective related to effective communication, which one would expect representatives of industry to consider extremely important, received rankings ranging from one through nine. Such a wide variation from such a small group (there were 14 members in the group), and indeed, not a random group but what one would assume was a fairly homogeneous group, is troubling.

We adopted the following approach in our program: A small group of faculty drafted a preliminary set of objectives. This draft was discussed by the Undergraduate Studies Committee of the department which included a student representative, and revised appropriately. This was then circulated electronically to all the faculty members for their comment and feedback. The objectives were slightly revised based on this feedback and this version was posted on electronic student newsgroups for discussion and comment. This version was also presented to our industrial advisory group at a meeting for their comment. A final version was then drafted and approved by faculty after further discussion at a faculty meeting. The final approved version certainly differed from the preliminary draft, but the essential ideas were the same as in that draft. (I will present some of our objectives in the next section; the complete set is available from our web site [3].)

Criterion 2 also requires us to periodically evaluate the objectives based on constituent needs. Since the objectives are published on our web site as part of our on-line program brochure, current students have the opportunity to comment on them at any time. In addition, each year we send out a survey to alumni who graduated from the program either two or six years ago ; part of the survey asks the respondent to rank, on a scale of Extremely Important to Not Important, the importance of each of our objectives with respect to their professional employment. Another similar survey is sent to a group of people in industry who,

\footnotetext{
${ }^{2}$ This ensures that we do not ask the same alumni every year which, we hope, will ensure reasonable response rates and will also give us, over the years, feedback from a broader group of alumni. Since our program is relatively large, producing about 100 graduates annually, this is a reasonable option.
} 
we believe, are likely to be in supervisory positions over our recent graduates; this survey again asks the respondent to rank, on a scale of Extremely Important to Not Important, each of our objectives with respect to its value for their particular organization.

The results of these surveys (also available at our web site) are mixed, but generally positive. Survey respondents generally seem to approve of our objectives though perhaps they would reduce some of the stress on conceptual curricular matters and increase the stress on more business oriented skills such as communication abilities. Our current students also seem to generally approve of our objectives although they too would probably like to see us shift some of our focus to more immediately marketable skills such as experience with specific software products. In the next year or so, the department's undergraduate studies committee will analyze the responses and see what changes, if any, need to be made.

A literal reading of the criteria may raise some question as to whether our approach meets the requirements. In particular, since we did not use input from alumni or from employers in arriving at our initial set of objectives, and since these two groups are clearly two of our constituencies, one might claim that we did not "determine our objectives based on the needs of [our] constituencies". I believe this is not a valid concern. Faculty do not operate in a vacuum; for example, they interact with on-campus recruiters as well as other industry people; alumni too provide faculty with input in informal interactions. Thus even though the original set of objectives might have been drafted by a small group of faculty, and even though this group did not seek inputs via surveys or other formal instruments before preparing the draft, given that the people in this group were senior, responsible, faculty in the department, we can be fairly certain that the objectives they came up with would not only meet our constituencies' needs, but also help advance the state of the art in computer science and engineering. If this is correct, I think it would be useful to revise the criteria to allow, and even encourage, programs to depend on the judgment of senior faculty in arriving at suitable objectives for the program and not simply adopt whatever objectives receive the highest numerical rankings in anonymous surveys; this will help guard against the type of problem revealed by Sener's [9] work and that seems to be common with surveys.

\section{Objectives and Outcomes}

Two approaches seem to be commonly used by engineering programs in their objectives and outcomes. The first approach, see [7] for an example, is to directly base the program objectives on the abilities 3.a through 3.k listed in Criterion 3; to these one would add some discipline-specific items, since Criterion 3 abilities are all general engineering abilities. The clear advantages of this approach are that Criterion 3 provides a ready-made, well-thoughtout list of items to include in the objectives. Moreover, demonstrating that graduates of the program meet its objectives will imply that they also have the 3.a through 3.k abilities which, in any case, has to be shown. The disadvantage is that it clutters up the statement of high-level program objectives with too much detail; moreover, Criterion 3 applies across all engineering disciplines, so many of the items may require repetition in discipline-specific terms. 
In our Computer Science and Engineering (CSE) program we used the alternate approach of identifying high-level objectives that captured our key goals. Thus, the first objective of our program [3] is:

To provide graduates with a thorough grounding in the key principles and practices of computing, and in the basic engineering, mathematical, and scientific principles that underpin them.

The other three objectives of our program are concerned respectively with, general engineering -as against $C S E$ - principles and the mathematical and scientific concepts underpinning them; the overall human context in which engineering activities in general and computing activities in particular, take place; and the specific goal of preparing graduates for employment in the CSE profession and for admission to graduate programs.

For each of these objectives, we identified specific corresponding outcomes. For example, one of the outcomes corresponding to our first objective reads:

[Students will] demonstrate proficiency in relevant aspects of mathematics, including discrete mathematics, as well as the appropriate concepts from physics and electrical circuits and devices.

This outcome not only corresponds to the objective quoted above, but is also directly related to the Criterion 3.a outcome [ability to apply knowledge of mathematics, science, and engineering] and to a somewhat lesser extent to 3.b [ability to design and conduct experiments and to analyze and interpret data], 3.c [ability to design a system or process to meet desired needs], and 3.e [ability to identify, formulate, and solve engineering problems]. Another outcome, related to the objective that has to do with the human context of engineering and computing activities, reads:

[Students will] obtain familiarity with basic ideas and contemporary issues in the social sciences and humanities.

This outcome is directly related to the Criteria 3.h outcome [ability to understand the impact of engineering solutions in a global/societal context] and to a lesser extent with 3.j [knowledge of contemporary issues]. Thus, although our objectives and outcomes were designed to accurately describe the goals of our program, our outcomes do correspond naturally (if not on a one-to-one basis) to all of the Criterion 3 outcomes. Thus, as in the case of the other approach, demonstrating that our graduates meet the objectives and outcomes of our program will imply that they also meet the Criterion 3 outcomes.

Before concluding this section, I would like to discuss one important point concerning program objectives and outcomes. It seems to me that the objective I have quoted above, or something very similar to it, would be appropriate for almost every program in Computer Science and Engineering. Indeed, if the word 'computing' in the objective were replaced by another discipline 'XYZ', the objective would be appropriate for nearly all programs in 'XYZengineering'. Again, our objective that has to do with preparing students for employment in the CSE profession, would seem appropriate for every program in CSE; and if 'CSE' were replaced by some other discipline 'XYZ', such an objective would be appropriate for nearly all programs in 'XYZ-engineering'. Similarly, the outcome I have quoted above concerning 
proficiency in relevant aspects of mathematics etc., would, I think, be entirely appropriate for nearly every program in CSE. And the outcome concerning ideas and issues in the social sciences, I think, is appropriate for all engineering programs.

Given this, it would seem very useful if a standard set of program objectives and outcomes were developed for each program area and published as part of the (program-specific portion of) EC 2000. Programs would not be required to adopt these but they could at least serve as starting points that individual programs could base their own objectives and outcomes on. This would allow programs to learn from each other, rather than each program starting from scratch. The intention in EC 2000, behind allowing each program to determine its own objectives and outcomes, was presumably to allow for some flexibility to individual programs. But instead, it seems to have evolved into the burdensome requirement that each program must come up with its own unique set of objectives and outcomes. Engineering programs in general, and engineering programs in specific disciplines in particular, have far more in common with each other than they have differences. Insisting that each program identify a unique set of objectives and outcomes on the basis of an involved process of gathering inputs from various constituents etc., will, it seems to me, exaggerate the differences and ignore the commonalities; it is also fraught with difficulties such as those identified by Sener [9] and Aldridge and Benefield [2] that I briefly mentioned in Section 2. Providing, as I have suggested above, a common set of objectives and outcomes for each engineering discipline that programs in that discipline could use, or at least use as a starting point, would help address this potentially serious problem.

\section{Assessment and Feedback Mechanisms}

We use the following assessment mechanisms in our program:

1. Assignments and examinations: Assignments, including programming projects where appropriate, and examinations, both mid-term and final, are standard in all courses.

2. Student Evaluations of Teaching (SETs): SETs, administered near the end of each term, allow students to provide the instructor (anonymous) feedback on the effectiveness of the course as taught by that particular instructor.

3. Exit surveys: All students complete an exit survey about two quarters prior to graduation. The survey allows the student to rank, for each of our objectives and outcomes, how well the program met that objective or outcome as well as the importance of the objective or outcome.

4. Alumni surveys: These are similar to the exit surveys and allow the respondent to rank the importance of each of our objectives and outcomes in their current professional position, and rank how well they felt they were prepared by the program with respect to that objective or outcome.

5. Supervisor/Manager surveys: This is similar to the alumni survey; the respondent is asked to rank the importance of each of our objectives and outcomes and how well the program prepared the respondent's recent supervisees with respect to each. 
Assessment mechanisms like these seem fairly standard and many programs use them. But assessment is not, of course, the end-goal. The end-goal is to use the data gathered from the assessments to arrive at possible improvements in the program. Thus Criterion 2 reads, in part, "[The program must] use the results [of the ongoing evaluation] to improve the effectiveness of the program." Similarly, Criterion 3 requires, "Evidence must be given that the results [of the assessment process] are applied to the further development and improvement of the program." Presenting such evidence can be quite a challenge; Lohmann [5] notes, "Rarely are curriculum changes and other major academic policy shifts the result of a single survey, one meeting of an advisory board, etc. They are typically the result of input from many sources, often over a period of time, and frequently involve many faculty meetings and committees. As such, ... document[ing] the connection from a given result to a specific change was very difficult." And indeed, I would add, many of the improvements in individual courses tend to be triggered by spontaneous discussions between various faculty and between faculty and students in hallways and classrooms, rather than as a result of inputs gathered via formal instruments such as surveys.

In order to deal with the dual challenges of using the results of our assessments to improve the program and of documenting this, we implemented a mechanism that we call Course Group Reports (CGRs). The entire curriculum is organized into seven or eight groups of related courses. One group, for example, is the Software Engineering group; another is Programming Languages; etc. Once every two years, the faculty coordinators of the courses in a given group are responsible for producing a report, the $C G R$, and presenting it to the department's Curriculum Committee. The report is expected to address such questions as whether the courses in the group are meeting their objectives, whether prerequisites are appropriate, suitability of the current textbooks, student reactions to the courses, relation of the group to the rest of the program, and whether the courses in the group are helping meet the overall program objectives as intended. The CGR also details the group's ideas for possible changes in the courses and explain how these changes will improve the courses in the group, as well as the program as a whole. In all of this, the group is expected to draw upon (and document in the CGR) the inputs obtained from the various assessment mechanisms I listed above, as well inputs that may have been obtained by other possibly ad-hoc means. For example, the report from the Programming Languages Group notes the feedback from students, and alumni, as well as from on-campus recruiters, concerning the importance of providing opportunities to our students to learn recent languages such as Perl and Python; (partly) in response to this, we are currently in the process of developing a course that will include fairly extensive coverage of Perl.

The CGR mechanism has proved extremely useful. First, it allows us to summarize the data collected from our various assessment mechanisms and present them in coherent contexts of groups of related courses, rather than as tables of raw numbers. Second, it enables faculty who are not directly involved with courses in a given group $G$ but who are still interested in $G$, perhaps because the courses they teach have, as prerequisites, one or more of the courses in $G$, to get a full understanding of the courses in $G$ and make any needed adjustments to their own courses. Third, it allows new faculty to acquire a thorough understanding of the various courses in the curriculum by perusing recent CGRs of relevant 
groups. Fourth, it ensures that faculty in related courses make it a point to interact with each other on a regular basis, when preparing the CGR for the particular group. And finally, the CGRs provide the evidence called for by EC 2000 to show how we use the assessment results to improve the effectiveness of the program. I believe a mechanism like this helps enormously in meeting this EC 2000 requirement and in addressing the question, quoted above, that Lohmann [5] raises; most programs will, I believe, find such a mechanism worth implementing. Recent CGRs from several different groups in our program are available at our web site.

Let me return to the assessment mechanisms. In our experience, by far the most useful assessment mechanisms have been assignments and examinations in individual courses. These time-tested tools provide immediate and valuable feedback to both the instructor and the student. They also allow the instructor to identify any potential problem in related courses; for example, if the performance of a large number of students in a given assignment or examination seems to indicate that they do not understand a concept they should have acquired in a prerequisite course, that probably indicates a problem with that course. For example, the instructors of our course on databases had noticed, based on student performance in assignments and examinations in the course, that students seemed to have difficulty with formal assertions, especially those that involved quantifiers; students should have become familiar with quantifiers in the discrete mathematics course (which is a prerequisite to the database course) but apparently this was not happening; to fix the problem, the database instructors suggested changes to the discrete mathematics course to the coordinator for that course, and the situation has improved. By contrast, other mechanisms such as our exit survey or alumni survey have not provided us with as much useful information.

This seems to be the experience of other programs as well. Especially compelling in this respect is the work of Addington and Johnson [1]. They report the results obtained from two sets of assessments used in their EE program at Virginia Military Institute (VMI). The first set consists of student surveys similar to, but more extensive than our exit surveys; students are asked to rank not only how well all the EE courses as a whole contributed to meeting the program's sixteen outcomes but also rank for each EE course, how it contributed toward each of the outcomes; in each ranking, students are asked to evaluate along three distinct axes: quality of opportunity, quantity of opportunity, and actual achievement. The second set provides similar assessment of the EE courses, but from the point of view of the faculty who teach the courses; this assessment is obtained by having faculty record (in a central database) their evaluation of how much each assignment in their particular course contributes to each of the sixteen outcomes; the 'achievement' component is evaluated on the basis of average student performance in the assignments.

The differences between the results from the two sets of assessments are striking. For example, for all the EE courses as a whole, the faculty's assessment of the amount of opportunity provided for the various outcomes ranged from under 0.5 to over 4.5 on a $0-5$ scale; one would expect such variation since EE courses would not contribute as much to, say, 'knowledge of contemporary issues' as to items directly related to the discipline. But no such variation appears in the student survey; in that survey, the corresponding range is from 3.0 to 3.9 (on a $1-5$ scale). The result for individual courses is similar. For example, the 
course (EE 362) that the student survey ranks as providing the most opportunity for achieving outcome 1, is ranked, in the faculty's evaluation, substantially below average among the various courses in doing this. If we assume, as seems reasonable, that the evaluation of faculty teaching the courses in question is reliable, then this suggest that exit surveys and, by extension, alumni surveys, may not be entirely reliable and should be used with care.

The Joint Task Force on Engineering Education Assessment, in its report [6], notes this problem. The report cautions "Most, if not all, measures available are limited in their ability to assess program quality. ... Until the results of these studies [validating the relationships of measures to actual professional performance] are available, actions taken to improve programs on the basis of such measurements would be both premature and ill-advised." In our experience, this caution is particularly applicable to surveys and other similar instruments, whereas assignments and examinations in individual courses provide more reliable and useful (as well as immediate) feedback to both students and instructors. Unfortunately, EC 2000 seems to play down the importance of these time tested assessment tools; I believe it would be useful to mention, indeed stress, in Criterion 3, the important role that assignments and examinations in individual courses can play in the overall assessment process.

\section{Discussion}

My goals in this paper were twofold. First, I wished to present some of the experiences of my program in preparing for accreditation evaluation under EC 2000, since this might help other programs preparing for evaluation in the near future. The main points here may be summarized as follows:

1. Constituent inputs: In seeking and utilizing input from constituents, especially with respect to program objectives and outcomes, it is important to recognize that obtaining input from industrial representatives, and even from alumni, tends to be difficult, sample sizes tend to be small, and the range of opinions expressed tends to be large. Hence, we found it advisable to depend heavily on faculty (especially senior faculty's) judgment in arriving at least at the initial draft of objectives and outcomes.

2. Objectives and outcomes: We found it best to draft the program objectives in such a way that they provided a high-level description of the main goals of the program (rather than try to model them after Criterion 3 outcomes); we then drafted the outcomes to provide details of specific abilities that we expected our students to acquire in the program. We found that each of the Criterion 3 outcomes mapped to one or more of our outcomes, with only minor tweaking of the latter. I believe most programs will find this approach useful.

3. Assessment mechanisms: We found that standard assignments and examinations in individual courses are extremely good assessment tools for the students, for the instructor, and for the program as a whole; other tools such as exit surveys were useful but results from these mechanisms are not entirely reliable. The experience of other programs seems similar. 
4. Feedback mechanisms: Our approach of organizing all the courses in the program into a handful of related groups, and having faculty involved with the courses in a group prepare a comprehensive report addressing a range of questions from the adequacy of prerequisite courses to how well the courses were helping to meet program objectives and outcomes as intended, has numerous advantages. It also provides documentation, called for by EC 2000, of how the program uses the results of the assessments to improve the effectiveness of the program.

The second goal of this paper was to suggest, based on the experiences of our program as well as those of other programs as reported in the literature, some possible refinements and modifications to EC 2000. The suggestions I have motivated and discussed in the paper may be summarized as follows:

1. Refine Criterion 2 to make sure that it is appropriate to depend upon faculty judgment in drafting objectives and outcomes; among other things, this will make it possible to take account of inputs that may have been obtained from constituents in informal settings (rather than depending exclusively on formal mechanisms for such inputs).

2. Develop and publish standard objectives and outcomes for each engineering discipline (perhaps as part of Criterion 8) and make it clear that these would be appropriate at least as starting points for programs to use for their objectives and outcomes statements; this will allow programs to learn from each other, rather than each program having to start from scratch.

3. Assessment mechanisms: Stress the importance of examinations and assignments in individual courses for assessment of both the individual courses as well as the program as a whole; as it is, both the criteria and the assessment-related literature, focus heavily on other mechanisms such as exit surveys, alumni surveys etc., but the reliability of these instruments is somewhat unclear.

\section{References}

[1] J. Addington and R. Johnson. Incorporating the design and use of surveys with other engineering assessment methods under Criteria 2000 guidelines. In Proceedings of ASEE Annual Conference. American Society for Eng. Education, 1999.

[2] M.D. Aldridge and L.D. Benefield. Assessing a specific program. In How do you measure success, pages 27-34. ASEE Professional Books, 1998.

[3] CIS Department. Undergraduate programs. http://www.cis.ohio-state.edu/AcademicPrograms/Undergraduate/index.html.

[4] Accreditation Board for Engineering and Technology. Engineering Criteria 2000. In How do you measure success, pages 13-16. ASEE Professional Books, 1998.

[5] J. Lohmann. EC2000: The Georgia Tech Experience. Journal of Engineering Education, pages 305-310, July 1999. 
[6] Joint Task Force on Eng. Education Assessment. A general assessment framework. In How do you measure success, pages 13-16. ASEE Professional Books, 1998.

[7] M. Parten and J. Bredeson. Implementing ABET 2000: An example work in progress. In Proceedings of ASEE Annual Conference. American Society for Eng. Education, 1999.

[8] G. Peterson. A bold new change agent. In How do you measure success, pages 5-10. ASEE Professional Books, 1998.

[9] E.M. Sener. Incorporating industrial advisory boards into the assessment process. In Proceedings of ASEE Annual Conference. American Society for Eng. Education, 1999. 